\title{
Global aspects of gravitomagnetism
}

\author{
A. Barros $^{1}$, V. B. Bezerra ${ }^{2}$ and C. Romero ${ }^{2}$ \\ ${ }^{1}$ Departamento de Física, Universidade Federal de Roraima, \\ 69310-270, Boa Vista, RR, Brazil \\ ${ }^{2}$ Departamento de Física, Universidade Federal da Paraíba, Caixa \\ Postal 5008, 58051-970, João Pessoa, PB, Brazil \\ e-mail: cromero@fisica.ufpb.br
}

Typeset using REVTEX 


\begin{abstract}
We consider global properties of gravitomagnetism by investigating the gravitomagnetic field of a rotating cosmic string. We show that although the gravitomagnetic field produced by such a configuration of matter vanishes locally, it can be detected globally. In this context we discuss the gravitational analogue of the Aharonov-Bohm effect.
\end{abstract}

PACS: 04.20.Cv; 95.30.S

Keywords: Gravitomagnetism; Spinning Cosmic String; Global aspects

\title{
I. INTRODUCTION
}

The conjecture that mass currents should generate a field called, by analogy with eletromagnetism, the gravitomagnetic field, goes back to the beginnings of general relativity [1]. Indeed, according to general relativity, moving or rotating matter should produce a contribution to the gravitational field that is the analogue of the magnetic field of a moving charge or magnetic dipole. This field would be expected to manifest itself in a number of effects, such as the Lense-Thirring precession [2], the gravitomagnetic time delay [3], change in the phase of electromagnetic waves [4], among others.

Recently, interest in the subject has been boosted by the concrete possibility that gravitomagnetic effects might be measured with the current technology of laser ranged satellites (LAGEOS and LAGEOS II) [5]. It is important to mention the Relativity Gyroscope Experiment (Gravity Probe B), a space mission to be launched this year whose aim is to detect gravitomagnetism effects directly. It is expected that these experimental programs will open new possibilities of testing general relativity and other metric theories of gravity $[6,7]$.

In the usual approach to gravitomagnetism one generally assumes the weak field and slow motion approximation of general relativity. It is in this limit that gravity parallels electromagnetism. On the other hand, interesting attempts to give a precise characterization 
of gravitomagnetism, which does not depend on the linearization of general relativity, have been made. For instance, an invariant characterization of gravitomagnetism (valid for any metric theory) has been proposed, which uses the pseudoscalar ${ }^{*} R R \equiv \frac{1}{2} \epsilon^{\alpha \beta \sigma \rho} R_{\sigma \rho}^{\ldots \nu} R_{\alpha \beta \mu \nu}$ as a measure of the presence of gravitomagnetism in a given spacetime [8]. However, since curvature is a local concept, this characterization would not work if the gravitational field manifest itself only globally, as in the case of spacetimes generated by cosmic strings. Therefore it seems to be of interest to develop a way of tackling such special cases. Clearly, a technique which seems to be quite appropriate to investigate global aspects of gravitomagnetism is holonomy theory, and, in fact, it has been employed recently to investigate the holonomic manifestation of the gravitomagnetic clock effect [9].

This paper is organized as follows. In Section II, we introduce the reader to the basic ideas of local gravitomagnetism. In Section III, we consider the spacetime generated by a spinning cosmic string and then investigate the gravitomagnetism associated with this kind of matter configuration. The Aharonov-Bohm effect in connection with the gravitomagnetism will be examined in Section IV. Finally, Section V is devoted to our conclusion and some remarks.

\section{THE GRAVITOMAGNETIC FIELD IN GENERAL RELATIVITY}

Let us recall that in the weak field approximation of general relativity we assume that the metric tensor $g_{\mu \nu}$ deviates only slightly from the flat spacetime metric tensor. In other words, we assume that $g_{\mu \nu}=\eta_{\mu \nu}+h_{\mu \nu}$, where $\eta_{\mu \nu}=\operatorname{diag}(-1,1,1,1)$ denotes Minkowski metric tensor and $h_{\mu \nu}$ is a small perturbation term. Then, by keeping only first-order terms

in $h_{\mu \nu}$ and adopting the usual harmonic coordinate gauge $\left(h_{v}^{\mu}-\frac{1}{2} \delta_{\nu}^{\mu} h\right)_{, \mu}=0$, the Einstein equations become

$$
\square \bar{h}_{\mu \nu}=-\frac{16 \pi G}{c^{4}} T_{\mu \nu}
$$

where $\bar{h}_{\nu}^{\mu}=h_{v}^{\mu}-\frac{1}{2} \delta_{\nu}^{\mu} h$ and $h$ denotes the trace of $h_{v}^{\mu}[10]$.

We now assume a perfect fluid matter configuration and slow motion. If $\sigma$ denotes the mass density and $v_{i}$ the velocity components, then (1) yields 


$$
\begin{aligned}
& \square \bar{h}_{00}=-\frac{16 \pi G}{c^{2}} \sigma \\
& \square \bar{h}_{0 i}=\frac{16 \pi G}{c^{3}} \sigma v_{i}
\end{aligned}
$$

where terms such as $p$ and $v_{i} v_{j} / c^{4}$ have been neglected. Let us now specialize the equations above to the case of a stationary gravitational field of a slowly rotating body. Then, far from the source we have

$$
\begin{gathered}
\nabla^{2}\left(\frac{c^{2} \bar{h}_{00}}{4}\right) \equiv \nabla^{2}\left(\Phi_{g}\right)=-4 \pi G \sigma \\
\nabla^{2} \bar{h}_{0 i}=\frac{16 \pi G}{c^{3}} \sigma v_{i}
\end{gathered}
$$

from which it follows that

$$
\begin{gathered}
\Phi_{g}=\frac{G M}{r} \\
\vec{h}=-\frac{2 G(\vec{J} \times \vec{r})}{c^{3} r^{3}} \equiv-\frac{2 \vec{A}_{g}}{c^{2}}
\end{gathered}
$$

where $\bar{h}_{0 i}$ are the components of the vector $\vec{h}, M$ and $\vec{J}$ are the total mass and angular momentum of the source, respectively. In close analogy with electrodynamics we define the gravitoelectric field to be $\vec{E}_{g}=-\nabla \Phi_{g}$ and the gravitomagnetic field to be $\vec{B}_{g}=\vec{\nabla} \times \vec{A}_{g}$. It is interesting to see that the condition $\bar{h}_{, \mu}^{\mu \nu}=0$ leads to $\nabla \cdot \vec{A}_{g}=0$ (analogous to the Coulomb gauge of electromagnetism).

Let us note that for the case of a slowly rotating sphere with angular momentum $\vec{J}=$ $(0,0, J)$, we obtain from $(7)$ in spherical coordinates

$$
\bar{h}_{0 \varphi}=h_{0 \varphi}=-\frac{2 J G}{r c^{3}} \sin ^{2} \theta
$$

Recalling that the Kerr metric in Boyer-Lindquist coordinates in the weak field and slow motion limit is given by [11] 


$$
d s^{2}=-\left(1-\frac{2 M G}{r c^{2}}\right) c^{2} d t^{2}+\left(1+\frac{2 M G}{r c^{2}}\right) d r^{2}+r^{2}\left(d \theta^{2}+\sin ^{2} \theta d \varphi^{2}\right)-\frac{4 J G}{r c^{3}} \sin ^{2} \theta c d t d \varphi
$$

we see that $\bar{h}_{0 \varphi}$ is the $g_{0 \varphi}$ component of $(9)$.

It is worth noting that one can easily show by using the geodesic equation

$$
\frac{d^{2} x^{\mu}}{d s^{2}}+\Gamma_{\alpha \beta}^{\mu} \frac{d x^{\alpha}}{d s} \frac{d x^{\beta}}{d s}=0
$$

in the slow motion and weak field approximation, that [12]

$$
\frac{d^{2} \vec{r}}{d t^{2}} \cong\left(\overrightarrow{E_{g}}+\frac{2}{c} \frac{d \vec{r}}{d t} \times \vec{B}_{g}\right)
$$

From the above we see that both the gravitoelectric and the gravitomagnetic field are essentially local physical entities. It turns out, however, that nonlocal properties of gravitomagnetism may appear, for example, when we consider the spacetime generated by a spinning cosmic string. Let us consider this case in the next section.

\section{THE GRAVITOMAGNETISM OF A SPINNING COSMIC STRING}

As objects whose existence in the early Universe has been predicted by GUT theories, cosmic strings have attracted a lot of interest in the last two decades [13]. It was shown by Vilenkin that, according to general relativity, the spacetime generated by a static cosmic string has a conical singularity [14-16]. It is this conicity that gives rise to a series of notable gravitational effects, mainly in connection with the fact that the geometry is locally

flat albeit it possesses nontrivial global properties. Solutions corresponding to spinning cosmic strings were also found [17-20]. It has been shown that, in addition to the conical topology of the static string, the geometry of a spinning cosmic string has a helical time structure which allows for the existence of closed timelike curves near the source [21], as well as gravitational time delay effects [22]. On the other hand, the gravitational time delay in ray propagation due to rotating bodies has been shown recently to be an effect directly 
related to the gravitomagnetic field produced by the bodies [3], which is rather suggestive of a possible presence of gravitomagnetic effects caused by a spinning cosmic string.

The spacetime of a spinning cosmic string is described by the metric [17]

$$
d s^{2}=-\left(c d t+\frac{4 G J}{c^{3}} d \phi\right)^{2}+d \rho^{2}+a^{2} \rho^{2} d \phi^{2}+d z^{2}
$$

where $a=1-\frac{4 G \mu}{c^{2}}$ is a measure of the angle deficit and $J$ denotes the angular momentum per unit of length of the string. This solution was obtained by a lifting from the $(2+1)$ dimensional spinning particle solution $[23]$ to $(3+1)$ dimensions. This was performed by simply adding $d z^{2}$ to the metric of the spinning particle spacetime. It is worth noting that the spacetime of a spinning cosmic string is locally flat everywhere except at the source. This is immediately seen by carrying out the coordinate transformation

$$
t^{\prime}=t+4 \frac{G J}{c^{4}} \phi, \quad \phi^{\prime}=a \phi, \quad \rho^{\prime}=\rho, z^{\prime}=z
$$

Note that if $J=0,(12)$ represents the metric $\gamma_{\mu \nu}$ of the spacetime generated by a static vacuum cosmic string [14-16]. If we consider low rates of spin motion, then the metric (12) may be written as

$$
d s^{2}=-c^{2} d t^{2}+d \rho^{2}+a^{2} \rho^{2} d \phi^{2}+d z^{2}-\frac{8 G J}{c^{2}} d t d \phi
$$

Thus, we may view the line element above as a perturbation of the metric of the static cosmic string spacetime, i.e. it has the form $d s^{2}=g_{\mu \nu} d x^{\mu} d x^{\nu}$, with $g_{\mu \nu}=\gamma_{\mu \nu}+h_{\mu v}$, where $h_{\mu v}$ is to be regarded as a small perturbation of $\gamma_{\mu \nu}$. Thus, in the same way as one can introduce the gravitomagnetic field directly from the Kerr metric in the weak field and slow motion approximation [1], it seems natural to define the gravitomagnetic vector potential by

$$
\vec{A}_{g}=-\frac{c^{2}}{2} \vec{h}=-\frac{c^{2}}{2}\left(h_{0 \rho}, h_{0 \phi}, h_{0 z}\right)=\left(0, \frac{4 G J}{c}, 0\right)
$$

where $\vec{h} \equiv\left(h_{01}, h_{02}, h_{03}\right)$.

Therefore, from the above equation, we conclude that $\vec{B}_{g}=\vec{\nabla} \times \vec{A}_{g}=0$, i.e. the gravitomagnetic field vanishes locally. However, taking into account the nontrivial topology 
of the spacetime (12) one would wonder whether this quantity might somehow be detected globally. In the next section we shall show that, in fact, a global property of the gravitomagnetism associated with the spinning cosmic string is what is called the gravitational analogue of Aharonov-Bohm effect [24].

\section{A GRAVITATIONAL ANALOGUE OF THE AHARONOV-BOHM EFFECT}

Gravitational analogues of the Aharonov-Bohm effect have been studied by Dowker [25] and others [26,27], mainly in connection with the conical spacetime [28]. The essential requirement for the analogy is the existence of a spacetime whose curvature vanishes everywhere except in some localized region. In such spacetimes it is possible that particles travelling along paths where the curvature vanishes still feel a physical effect in much the same way as the wave function of a charged particle undergoes a change of phase

$$
\delta \alpha=\frac{e}{\hbar c} \oint_{C} \vec{A} \cdot d \vec{l}
$$

when the integration above is carried out around a closed circuit $C$ including a region where the magnetic field $\vec{B}$ is nonvanishing, even if the wave function is zero in this region, an effect which has no counterpart in classical physics and has been measured in a number of experiments [29].

Other effects analogous to the electromagnetic Aharonov-Bohm effect exist in a classical context, such as the Sagnac effect [30] in general relativity, which consists in a phase shift between two beams of light traversing in opposite directions the same path around a rotating mass distribution.

Now, let us turn our attention to the change of phase induced by the gravitomagnetic vector potential given by (14). Clearly, it is given by

$$
\delta \alpha_{g}=\kappa \oint_{C} \overrightarrow{A_{g}} \cdot d \vec{l}=\frac{8 \pi \kappa G J}{c}
$$

where we have introduced the constant $\kappa$ in order to ensure that $\delta \alpha_{g}$ is dimensionless. 
Therefore, we are led to the conclusion that although locally we have no gravitomagnetism, yet there is still a global effect, namely, a change on the phase, due to the gravitomagnetic potential, hence in close analogy with the Aharonov-Bohm effect. Incidentally, this result coincides with the holonomy [27] for general curves in the $x y$-plane, which enclose the spinning cosmic string, when $a=1$. A similar result can also be obtained from the Schrödinger equation governing the motion of a particle with electric charge $e$, moving in the field of an infinitely thin solenoid which carries a magnetic flux $\Phi$, when we replace

$\frac{e \Phi}{2 \pi}$ by $G J$. These results seems to suggest that the choice we have made for $\overrightarrow{A_{g}}$, given by (14), in the framework of the analogy between electromagnetism and gravitation, is correct.

\section{FINAL REMARKS}

Another global gravitomagnetic effect, which happens in the absence of the local gravitomagnetic field and is due to the spacetime topology only, is the time delay between the arrival time of two particles that describe arbitrary closed paths in opposite directions around a spinning cosmic string. It has been shown that this time delay, a gravitomagnetic observable effect at a classical level [22], is given by $\Delta \tau=\frac{16 \pi G J}{c^{4}}=\frac{4 \pi}{c^{4}} A_{g}$.

Gravitomagnetism is often regarded as a manifestation of the way inertia is incorporated into general relativity. According to this view, the measurement of gravitomagnetic effects would certainly be considered an experimental support of what is called the weak general relativistic interpretation of the Mach principle [1]. However, this interpretation is concerned with local inertial forces. The fact that gravitomagnetism may manifest itself also globally would suggest one to conjecture that the Mach principle may also possess a global character. 


\section{REFERENCES}

[1] I. Ciufolini, J. A. Wheeler, Gravitation and Inertia, Princeton University Press, Princeton $(1995)$.

[2] J. Lense, H. Thirring, Phys. Z. 19 (1918) 156 (English translation by B. Mashhoon, F.W. Hehl, D. S. Theiss, Gen. Rel. Grav. 16 (1984) 711.

[3] I. Ciufolini, S. Kopeikin, B. Mashhoon and F. Ricci, Phys. Lett. A 308 (2003) 101.

[4] J. M. Cohen and D. R. Brill, Nuovo Cim. B56 (1968) 209.

[5] I. Ciufolini, E. Pavlis, F. Chieppa, E. Fernandes-Vieira, J. Pérez-Mercader, Science, 279 (1998) 2100 .

[6] A. Camacho, Gen. Rel. Grav. 34 (2002) 1403.

[7] C. M. Will, Theory and experiment in gravitational physics, Cambridge University Press, Cambridge (1993).

[8] I. Ciufolini, Dragging of inertial frames, gravitomagnetism, and Mach's Principle, in Mach's Principle: From Newton's bucket to quantum gravity, edited by J. Barbour and H. Pfister, Birkhäuser, Boston (1995).

[9] R. Maarteens, B. Mashhoon and D. Matravers, Class. Quantum Grav. 19 (2002) 195.

[10] L. D. Landau and E. M. Lifshitz, The Classical Theory of Fields, Pergamon, Oxford $(1971)$

[11] R. H. Boyer, R. W. Lindquist, J. Math. Phys. 8 (1967) 265.

[12] B. Mashhoon, L. Iorio, H. Lichtenegger, Phys. Lett. A 292 (2001) 49

[13] A. Vilenkin ,E. P. Shellard,Cosmic Strings and Other Topological Defects, Cambridge University Press, Cambridge (1994).

[14] A. Vilenkin, Phys. Rev. D23 (1981) 852. 
[15] J. R. Gott, Astrophys. J. 288 (1985) 422

[16] W. A. Hiscock, Phys. Rev. D 31 (1985) 3288

[17] P. O. Mazur, Phys. Rev. Lett. 57 (1986) 929; 59 (1987) 2380.

[18] B. Jensen, H. Soleng, Phys. Rev. D45 (1992), 3528.

[19] J. P. S. Lemos, V. T. Zanchin, Phys. Rev. D53 (1996) 4684

[20] B. Jensen, Class. Quantum Grav. 9 (1992) L7.

[21] M. Novello, M. C. Motta da Silva, Phys. Rev. D49 (1994) 825.

[22] D. Harari, A. P. Polychronakos, Phys. Rev. D38 (1988) 3320.

[23] S. Deser, R. Jackiw and G. 't Hooft, Ann. Phys. (NY) 152 (1984) 220.

[24] Y. Aharonov, D. Bohm, Phys. Rev. 115 (1959)

[25] J. S. Dowker, Nuovo Cim. B52 (1967) 129.

[26] L. H. Ford and Vilenkin, J. Phys. A14 (1981) 2353; V. B. Bezerra, Phys. Rev. D35 (1987) 2031.

[27] V. B. Bezerra, Ann. Phys. 203 (1990) 392.

[28] L. Marder, Proc. Roy. Soc. A 252 (1959) 45.

[29] A. Tonomura et al., Phys. Rev. Lett. 56 (1986) 792; N. Osakabe et al., Phys. Rev. A34 (1986) 815; K. Kobayashi et al., J. Phys. Chem. Solids 63 (2002) 1301; S. Kasai et al., Appl. Phys. Lett. 81 (2002) 316; U. F. Keyser et al., Semicond. Sci. Techn. 17 (2002) L22.

[30] A. Ashtekar and A. Magnon, J. Math. Phys. 16 (1975) 341. 